\title{
Ex-vivo T-Cell Depletion
}

National Cancer Institute

\section{Source}

National Cancer Institute. Ex-vivo T-Cell Depletion. NCI Thesaurus. Code C155795.

Treatment that reduces or removes T cells from a graft before administration to the recipient. 